\title{
Asthénospermie immuno-induite par une protéine épididymaire, la préalbumine ovine (oPES)
}

\author{
S Fournier-Delpech, Y Guérin, M Magistrini, Y Combarnous
}

avec la collaboration technique de JJ Roi

CNRS-URA 1291, INRA, station de PRMD, 37380 Nouzilly, France

(Reçu le 27 septembre 1994; accepté le 10 mai 1995)

\begin{abstract}
Résumé - La motilité des spermatozoïdes éjaculés (\% de spermatozoïdes fléchants) a été observée au microscope optique chez 26 béliers ; la moitié des béliers a été immunisée à l'aide d'une protéine épididymaire sécrétoire (préalbumine épididymaire sécrétoire spécifique ou «PES» ovine) injectée selon des protocoles immunisants (adjuvant de Freund ou de Hunter) par voie soit intrapéritonéale soit intradermique (adjuvant de Hunter ou rappels de la protéine seule) ; l'autre moitié des béliers a reçu les mêmes produits à l'exception de la protéine (animaux témoins). II s'en suit une asthénospermie durant toute la durée du traitement, qui est contemporaine de la présence transitoire et de courte durée d'anticorps anti-PES dans le plasma séminal. Les béliers témoins n'ont pas développé ces anticorps. L'étude chez 64 brebis de la fertilité de 10 béliers immunisés qui ont recouvré une mobilité spermatique suffisante pour assurer la fécondation ne révèle pas de différences significatives avec celle des béliers témoins homologues.
\end{abstract}

bélier / immunisation / protéine épididymaire / spermatozoïde / fertilité

Summary - Autoimmune asthenospermia with a epididymal protein, prealbumin ovine (OPES). The motility of ejaculated spermatozoa (\% of progressive spermatozoa) has been evaluated visually on 26 adult rams. Half the animals were immunized with a secretory epididymal protein (prealbumin epididymal-specific (PES) ovine) injected according to immunizing protocols (Freund or Hunter adjuvant) intraperitoneally or intradermally (Freund or Hunter adjuvant) or intramuscularly (Hunter adjuvant or PES only). The other animals received the same product without the protein (controls). The product resulted in a strong asthenospermia which parallels the transient presence of anti-PES antibodies in the seminal plasma. A study with 64 ewes showed that the fertility of 10 immunized rams that had recovered sufficient forward motility to ensure fertilization, did not differ from that of control rams.

ram / immunization / epididymal protein / spermatozoa / fertility 


\section{INTRODUCTION}

Les spermatozoïdes testiculaires des mammifères sont inféconds ; ils acquièrent leurs compétences fonctionnelles (mobilité et pouvoir fécondant) dans l'épididyme sous la stimulation de la testostérone (Orgebin-Crist et al, 1975 ; Cooper, 1986). Plusieurs médiateurs du contrôle androgénique de la différenciation post-testiculaire des gamètes mâles sont des protéines sécrétées par l'épididyme qui se lient à des sites localisés sur des régions particulières de la membrane plasmique spermatique au niveau de la tête ou du flagelle (Cooper, 1986 ; Fournier-Delpech et al, 1987 ; Brooks, 1988). À ce processus, dont le mécanisme reste inconnu, sont associés chez le bélier des changements qualitatifs et quantitatifs de glycoconjugués de la surface spermatique (Fournier-Delpech et Courot, 1981 ; Hammerstedt et Parks, 1987 ; Dacheux et al, 1991). Ces modifications structurales sont concomitantes de l'acquisition du pouvoir fécondant et de compétences membranaires telles que la liaison à la zone pellucide et l'initiation de la mobilité progressive (Fournier-Delpech et al, 1979 ; Fournier-Delpech et Courot, 1987 ; Amann, 1987). Le présent travail a pour objet d'étudier, chez le bélier, les effets de l'immunisation à l'aide de l'une de ces protéines - la préalbumine épididymaire spécifique ovine ou oPES (Fournier-Delpech et al, 1987, 1988, 1990 ; Carles et al, 1992), sur la mobilité des spermatozoïdes éjaculés.

\section{ANIMAUX, MATÉRIEL ET MÉTHODES}

\section{Animaux, abréviations et produits}

\section{Animaux}

Nous avons utilisé des béliers de race Romanof choisis d'après la stabilité des caractéristiques de leurs éjaculats ; les expériences se sont dérou- lées de septembre à decembre. Vingt-six mâles âgés de 18 mois, entraînés à la collecte de sperme à l'aide d'un vagin artificiel, ont été répartis en 4 lots $(A=6, B=6, C=6, D=8$ animaux) chacun composé de 2 groupes égaux d'animaux soit immunisés avec la protéine $(n=3, n=3$, $n=3, n=4$, dans les lots $\mathrm{A}, \mathrm{B}, \mathrm{C}$ et $\mathrm{D}$, respectivement) soit témoins subissant le même traitement sans la protéine $\left(n^{\prime}=3, n^{\prime}=3, n^{\prime}=3, n^{\prime}=4\right.$, dans les lots $A, B, C$ et $D$, respectivement). Soixante-quatre femelles, âgées de 2 ans, ont été réparties en 3 lots $(E=24, F=20, G=20$ animaux), composés chacun pour moitié de brebis inséminées $56 \mathrm{~h}$ après l'injection de PMSG (250 Ul/brebis) pratiquée au moment du retrait du progestagène intravaginal $(30 \mathrm{mg} /$ brebis pendant 14 j), avec des spermatozoïdes soit de béliers témoins soit de béliers immunisés. L'OPES $a$ été purifiée à partir d'épididymes d'agneaux récupérés en abattoir.

\section{Abréviations}

Tampons : Tris- $\mathrm{HCl} 3 \mathrm{mM}, \mathrm{pH} 8,8,0,2 \%$ Azide ; PBS = phosphate physiologique sans calcium ; OPES = PES ovine $; \mathrm{PEG}=$ polyéthylène-glycol $4000 ;$ lgG-PO = IgG anti-mouton couplées avec la péroxydase de raifort ; OPD = orthophénylènediamine. $\mathrm{J}=$ jour ; sem $=$ semaine.

\section{Produits}

Ils provenaient de chez Tébu (Electrophorèse), Interchim (adjuvant de Hunter = Titer Max), Sigma (adjuvant de Freund et $F(a b)$ lgG-PO, OPD et Tween 20), CBM (plaques ELISA), Pharmacia (DEAE-Sephacel), Biorad (gélatine), Prolabo (autres produits).

\section{Méthodes}

\section{Préparation de l'antigène}

Les épididymes débarrassés des enveloppes conjonctives $(1 \mathrm{~kg})$ ont été congelés par lots de $1 \mathrm{~kg}$, broyés en Tris- $\mathrm{HCl}(1 \mathrm{l})$ et centrifugés (15 $\min , 10000 \mathrm{~g}, 4^{\circ} \mathrm{C}$ ). Le surnageant a été ajusté à $25 \mathrm{~g}$ de $P E G / 100 \mathrm{ml}$ et centrifugé à température ambiante. Le précipité a été dissous avec du tampon Tris- $\mathrm{HCl}$ additionné de Tween $(0,2 \%)$ passé sur une colonne de DEAE-Sephacel qui a été 
ensuite lavée avec du CINa à $20 \%$ dans du Tris$\mathrm{HCl}$; la fraction éluée par le $\mathrm{NaCl}$ à $30 \%$ a été dialysée contre du Tampon Tris-HCl puis soumise à une deuxième chromatographie sur DEAESéphacel, lavée avec du $\mathrm{NaCl}$ à $15 \%$ dans du tampon Tris- $\mathrm{HCl}$. La fraction $\mathrm{NaCl} 0,3 \mathrm{M}$ a été dialysée contre $\mathrm{H}_{2} \mathrm{O}$ et sa pureté a été vérifiée par électrophorèse (Fournier-Delpech et al, 1987 ; Carles et al, 1992). Elle a été alors utilisée pour les immunisations. Dix milligrammes ont été purifiés par électrophorèse préparative pour le dosage par compétition des anticorps anti-PES (voir cidessous).

\section{Traitement des béliers}

\section{Immunisations}

L'oPES a été administrée en émulsion (2 mg d'oPES/1 ml/injection) aux béliers immunisés à JO dans de l'adjuvant complet de Freund, puis à J8 et $\mathrm{J} 15$ dans de l'adjuvant incomplet soit par voie intrapéritonéale (lot A) soit par voie intradermique (lot B) en 10 points (face interne des cuisses). Les béliers immunisés (lot $\mathrm{C}$ ) ont reçu la protéine émulsionnée dans du Titer Max par voie intramusculaire aux jours $0,8,15,40,50,60$. De manière à prolonger, si possible, l'immunisation sans adjuvant, les béliers $D$ ont été traités comme les béliers $B$ puis par des injections d'oPES seule dans du PBS par voie intramusculaire aux jours $30,40,50,60,70$. Les béliers témoins ont été traités comme les béliers immunisés, mais sans oPES.

\section{Prélèvements}

Les prises de sang et collectes de sperme ont été effectuées tous les 5 à $10 \mathrm{j}$. Le sérum sanguin et le plasma séminal ont été obtenus par centrifugation et conservés à $-20^{\circ} \mathrm{C}$. Lors des prélèvements, les testicules et les épididymes ont été palpés en vue du dépistage clinique d'un éventuel processus inflammatoire.

\section{Étude des réponses biologiques}

\section{Réponse immunologique}

Les anticorps anti-oPES sont quantifiés sur plaques ELISA préalablement traitées par $0,5 \mu \mathrm{g}$ de PES/100 $\mu \mathrm{l} \mathrm{PBS/puits}\left(37^{\circ} \mathrm{C}, 1 \mathrm{~h}\right)$, et conservées 1 sem à $4^{\circ} \mathrm{C}$ puis successivement par la gélatine $\left(0,2 \%\right.$ dans du PBS, $\left.1 \mathrm{~h}, 37^{\circ} \mathrm{C}\right)$, PBS- gélatine à $0,2 \%$ (lavage), les sérums (1/2 000 à $1 / 5000)$ ou les plasmas séminaux $(1 / 25$ et $1 / 50)$ dilués dans du PBS-gélatine renfermant 0 à $10 \mu \mathrm{g}$ de PES $/ \mathrm{ml}$ et préalablement incubés $2 \mathrm{~h}$ à $37^{\circ} \mathrm{C}$, PBS $+0,2 \%$ de Tween) (lavage) ; ensuite, par les IgG-PO anti-mouton (1/3000 dans PBS-gélatine, $\left.1 \mathrm{~h}, 37^{\circ} \mathrm{C}\right)$, PBS-Tween (lavage), I'OPD (0,5 mg/ml tampon citrate d'ammonium, $\mathrm{pH} 5,6)$ en présence $\mathrm{d}^{\prime} \mathrm{H}_{2} \mathrm{O}_{2} 110 \mathrm{~V}(1 / 5000), \mathrm{SO} 4 \mathrm{H} 2(15 \%)$, lues à $792 \mathrm{mM}$ (Multiscan LKB). La concentration en IgG anti-PES est exprimée dans le sérum et dans le plasma séminal par, respectivement, le nombre de millimoles ou de micromoles moles saturantes de PES déduit de la courbe de compétition selon la méthode de Scatchard.

\section{Nombre et mobilité des spermatozoïdes}

La concentration des éjaculats en spermatozoïdes a été déterminée par densitométrie ; le pourcentage de spermatozoïdes fléchants a été estimé entre lame et lamelle sous le microscope en contraste de phase après dilution (300 millions de spermatozoïdes $/ \mathrm{ml}$ de lait écrémé), et incubation pendant $1 \mathrm{~h}$ à $37^{\circ} \mathrm{C}$.

\section{Fertilité et développement des agneaux}

La fertilité des béliers a été testée, après qu'ils ont recouvré 20 à $25 \%$ de formes fléchantes, soit par saillie naturelle (béliers A et femelles E), soit en insémination artificielle cervicale (béliers $B$ et $D$ et femelles $F$ et $G$ respectivement) ; 350 millions de $s p z$ /brebis, $56 \mathrm{~h}$ après l'administration de PMSG. La gestation a été diagnostiquée par le dosage de la progestérone plasmatique $18 \mathrm{j}$ après l'insémination ; les produits nés ont été pesés à 0 , 3,6 et 12 sem (lots $E$ et $F$ ) sans tenir compte des portées atypiques de plus de 4 agneaux (0 à 1 par lot expérimental).

Les résultats obtenus pour les différents groupes ont été comparés à l'aide du test de comparaison des moyennes (t de Student).

\section{RÉSULTATS}

\section{Appareil génital}

Les testicules et épididymes des béliers traités ou témoins n'ont présenté aucun signe clinique décelable d'inflammation. 


\section{La réponse immunitaire des béliers}

\section{Caractéristiques de l'antigène}

L'antigène purifié se présentait sous forme d'une seule bande d'électrophorèse conforme aux caractéristiques précédemment décrites (Foumier-Delpech et al, 1988). De plus, les homologies entre oPES et BSA ont fait l'objet d'un précédent travail (Carles et al, 1992).

\section{La réaction immunologique}

Elle a été mesurée par la quantité d'oPES saturante pour les anti-corps anti-PES présente dans les sérums et les plasmas séminaux des animaux immunisés. Elle était positive à partir de $\mathrm{J} / \mathrm{O}$ dans les sérums de tous les béliers immunisés $(0,05$ à $0,1 \mathrm{mM})$. Le taux maximal d'anti-PES est atteint à J25 chez les béliers immunisés A (4,7 \pm $2 \mathrm{mM}$ ) à J35 chez les béliers immunisés $B$ $(4,20 \pm 1,9 \mathrm{mM})$. En l'absence de rappel, les anticorps sont encore décelables à $\mathrm{J} 100$.

Dans le plasma séminal, la concentration d'anticorps du plasma est plus faible $(A=0,49 \pm 0,09 \mu \mathrm{M} ; \mathrm{B}: 0,51 \pm 0,2 \mu \mathrm{M})$ et de courte durée (dont le maximum est atteint 1 sem après la $3^{e}$ injection et qui est indécelable 3 sem plus tard). Elle est décelable $10 \mathrm{j}$ après une injection de rappel dans de l'adjuvant et présente les mêmes caractéristiques pour une même durée. Les plasmas séminaux des béliers immunisés qui ont produit les spermatozoïdes inséminés renfermaient tous des anti-PES de l'ordre de 0,5 à 1 micromole (A: $1,5 \pm 0,5 ; B: 0,8 \pm 0,6 ; D: 0,9 \pm 0,6$ ). Chez les animaux témoins, ni le sérum ni le plasma séminal ne renfermaient d'anti-PES décelables par dosage ELISA par compétition.

\section{Caractéristiques des spermatozoïdes éjaculés}

\section{Concentration}

La concentration en spermatozoïdes des éjaculats n'a présenté aucune différence significative en fonction ni du traitement ni de sa durée.

\section{Morphologie}

En l'absence de quantifications nous rapportons les faits les plus évidents au maximum de la réponse biologique, c'est-à-dire au minimum de la mobilité spermatique. Chez les béliers traités, 50 à $70 \%$ des spermatozoïdes étaient agglutinés 2 par 2 par la tête ; la gouttelette cytoplasmique était en position proximale. Chez les béliers témoins, les spermatozoïdes n'étaient pas agglutinés ; la gouttelette cytoplasmique était absente ou en position distale. Les flagelles étaient libres chez tous les animaux traités ou témoins.

\section{Mobilité des spermatozoïdes}

Chez les béliers traités par l'oPES l'observation de la figure 1 montre que le pourcentage de spermatozoïdes mobiles fléchants a diminué progressivement pour atteindre un minimum après environ $20 \mathrm{j}$ (lot $A$, injecté par voie intrapéritonéale) un peu plus tardivement dans les lots $B$ et $C$ (injecté par voie intradermique et intramusculaire, respectivement). Cette diminution a été transitoire car, après la fin du traitement, elle a été suivie d'une restauration dont la dynamique a été à peu près symétrique de celle de la diminution. Les pourcentages de spermatozoïdes fléchants ont été inférieurs à $20 \%$ à des moments variables selon la voie d'administration $\mathrm{J} 25$, 
Fig 1. Pourcentage de spermatozoïdes fléchants dans les éjaculats de béliers immunisés avec I'OPES ( $\square$ ) ou témoins $(\mathrm{O}-\mathrm{O})$. Éjaculats dilués dans du lait écrémé $(300 \mathrm{x}$ $10^{6}$ spermatozoïdes $/ \mathrm{ml}$ ). Les flèches indiquent les dates des injections soit de PES avec $(\rightarrow)$ ou sans $(\cdot>\rightarrow)$ adjuvant. Lot $A$ : béliers traités par l'adjuvant de Freund par voie intrapéritonéale ; lot $B$ : béliers traités par l'adjuvant de Freund par voie intradermique ; lot $C$ : béliers traités avec le Titer Max par voie intramusculaire ; lot $D$ : béliers traités comme ceux du lot $B$ puis avec soit la PES seule soit le soluté. I Déviation standard ; * différence significative $(P<0,05)$ entre béliers immunisés et béliers témoins pour un jour donné.

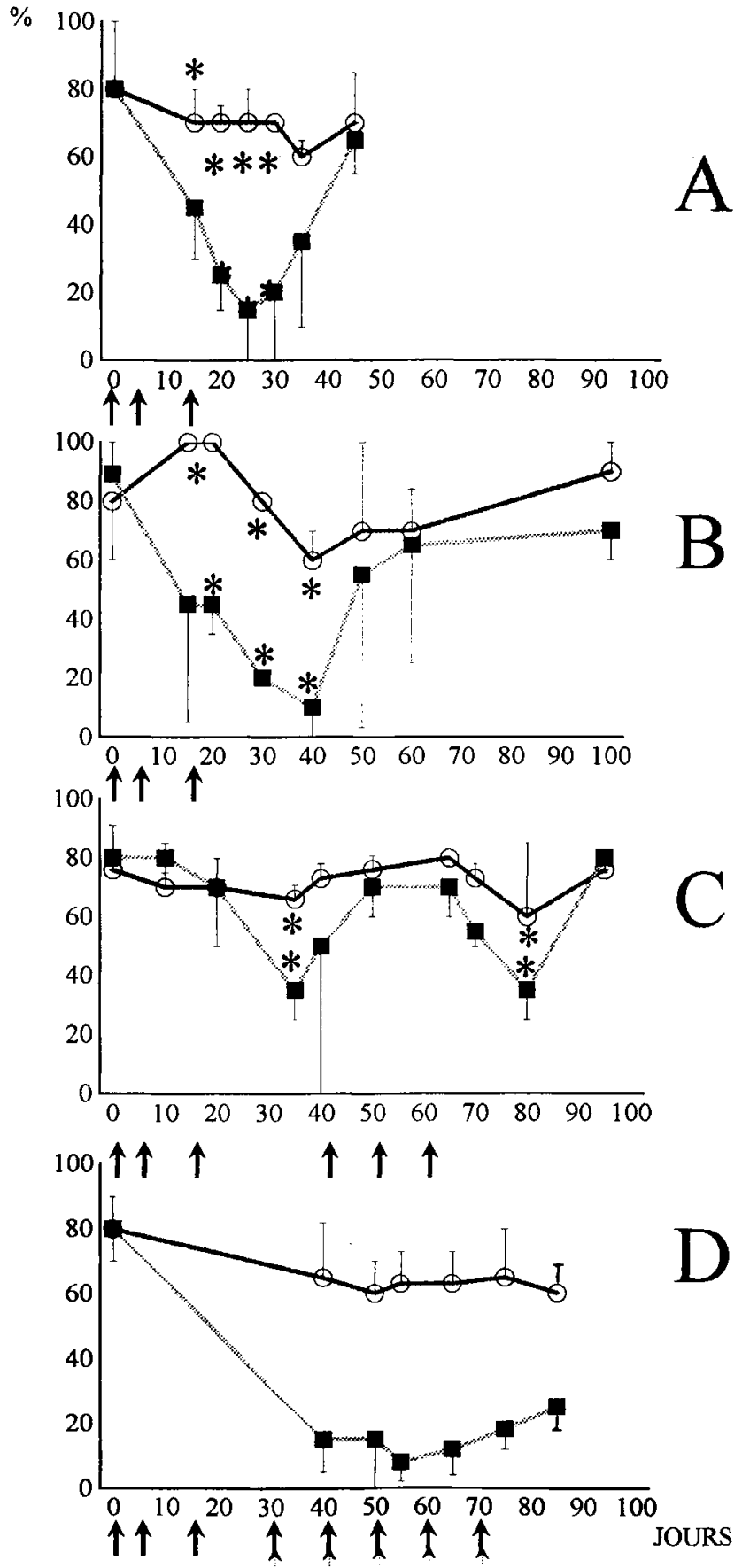


$\mathrm{J} 40$, à $\mathrm{J} 35$ et J80, pour respectivement les lots $\mathrm{A}, \mathrm{B}$ et $\mathrm{C}$; l'administration par la voie intrapéritonéale de la PES émulsionnée dans du Freund a produit des effets plus rapides et de moins longue durée que par la voie intradermique. L'immunisation à l'aide du Titer Max a été moins efficace que l'immunisation réalisée avec le Freund; en effet, le pourcentage de spermatozoïdes mobiles ne diminue pas audessous de $40 \%$. En l'absence de rappel, les spermatozoïdes ont recouvré progressivement leur mobilité en 2 sem. L'administration à J60 d'oPES dans de l'adjuvant a provoqué une nouvelle diminution de la mobilité qui a été rapide (car amorcée en 10 j) et de courte durée (béliers $\mathrm{C}$ ). Après induction de la réponse immunologique à l'aide d'adjuvant de Freund, I'asthénospermie (moins de 10\% de spermatozoïdes fléchants) a été maintenue pendant 1 mois par l'administration intramusculaire d'oPES seule, c'est-à-dire tant qu'a duré le traitement (Béliers immunisés D). II s'agit bien d'une réponse au traitement immunitaire, car significativement différente, de la diminution de mobilité fléchante due à l'adjuvant - Freund ou Titer Max - que I'on observe chez les témoins dont le pourcentage de formes animées d'une mobilité fléchante a diminué de moitié 20 à $30 \mathrm{j}$ après le début du traitement mais n'a jamais été inférieur à 40 .

\section{Fertilité des spermatozoïdes après récupération d'une mobilité fléchante}

Le tableau I montre que les descendances des béliers soit témoins soit immunisés n'ont pas présenté de différence significative dans la taille des mises bas, ni le poids aux différents stades du développement : naissance, période post-natale $(3,6$ et 12 sem).

Tableau I. Descendance mâle des béliers immunisés et ayant recouvré partiellement la motilité spermatique (25\% de SPZ fléchants).

Caractéristiques des $s p z$

Béliers Insémination

Lot Traitement

(5)

Adjuvant

Artificielle

$2,8 \pm 1,3$

$2,3 \pm 0,6(10)$

$6,4 \pm 1,4(8)$

$12,4 \pm 2,6(8)$

$25,0 \pm 4,36$

A (vaginale)

$$
\begin{aligned}
& \text { Adjuvant } \\
& + \text { PES }
\end{aligned}
$$

Adjuvant

$$
\text { Saillie }
$$

B

Adjuvant + PES
Poids des agneaux mâles (1re ligne) et femelles (2๑ ligne)

Naissance $\quad 3 \mathrm{sem} \quad 6 \mathrm{sem} \quad 12 \mathrm{sem}$

$2,8 \pm 0,8$

$2,2 \pm 1,1(7) \quad 5,9 \pm 1,8(5) \quad 10,1 \pm 3,4(5) \quad 18,7 \pm 4,1(5)$

$3,0 \pm 0,8$

$2,5 \pm 0,7(12)$

$5,7 \pm 1,2(11) \quad 11,7 \pm 2,4(11) \quad 22,4 \pm 4,3(9)$

$2,4 \pm 0,5(11) \quad 5,9 \pm 1,1(10) \quad 11,4 \pm 1,3(9) \quad 22,5 \pm 1,9(8)$

$2,7 \pm 0,5(12) \quad 7,2 \pm 1,0(8) \quad 13,4 \pm 1,5(8) \quad 25,0 \pm 2,2(8)$

$2,2 \pm 0,5(14) \quad 6,0 \pm 1,2(13) \quad 11,0 \pm 2,2(12) \quad 21,0 \pm 3,5(12)$

$3,1 \pm 1,0 \quad 2,6 \pm 0,6(14) \quad 5,7 \pm 1,2(12) \quad 11,7 \pm 2,4(12) \quad 24,4 \pm 3,5(12)$

$2,4 \pm 0,5(-12) \quad 5,9 \pm 1,1(12) \quad 11,3 \pm 1,3(10) \quad 21,5 \pm 2,8(10)$ 


\section{DISCUSSION}

Nous avons étudié sur les spermatozoïdes éjaculés de bélier l'effet de l'immunisation contre un auto-antigène sécrété par l'épithélium épididyrnaire : la préalbumine épididymaire ovine ou "OPES». Quand les spermatozoïdes parviennent dans l'épididyme, cette protéine se fixe sur la surface externe de leur membrane plasmique (tête et pièce intermédiaire) devenant ainsi un antigène spermatique post-testiculaire d'origine strictement épididymaire (Fournier-Delpech et al, 1987, 1988). Selon leur localisation sur les spermatozoïdes (tête ou flagelle), les antigènes spermatiques génèrent des immunoglobulines qui peuvent inhiber des fonctions nécessaires à la réalisation de la fécondation : telle, respectivement, la reconnaissance de l'ovocyte (Yanagimachi et al, 1981) ou la mobilité (Koyama et al, 1991). Des applications immunocontraceptives en découlent (Primakoff et Myles, 1990 ; Goldberg, 1990); elles sont limitées chez le mâle par les risques d'orchite qui peuvent survenir dans le cas d'antigènes spermatiques d'origine testiculaire (Pelletier et al, 1981). En principe, l'OPES ne comporte pas ce risque car elle est spécifique de l'épididyme (Fournier-Delpech et al, 1987). De fait, il semble que, dans nos expériences, l'immunisation à l'aide de l'oPES n'a pas provoqué d'orchite décelable ni altéré la production spermatique des béliers traités.

De telles protéines épididymaires sont des agents de la différenciation de la membrane spermatique (Jones, 1989 ; Cameo et al, 1990 ; Vreeburg et al, 1992). Produits de l'activité de gènes qui s'expriment spécifiquement dans l'épididyme (Brooks, 1988; Garrett et al, 1991 ; Carles et al, 1992), elles sont des médiateurs du contrôle hormonal qu'exerce la testostérone dans l'acquisition de la fertilité mâle (Orgebin-Crist et al, 1975). Elles ont été mises en évidence chez toutes les espèces de mammifères où elles ont été recherchées, y compris chez l'homme
(Jones et al, 1985 ; Cooper, 1986 ; Brooks, 1988). La plus étudiée est la PES du rat (connue aussi sous d'autres dénominations comme la «protéine D-E») (Fournier-Delpech et al, 1987), dont on a montré qu'elle subit une redistribution à la surface du spermatozoïde au cours de la capacitation (Rochwerger et Cuasnicu, 1992).

Leur potentiel contraceptif associé à leurs capacités auto-immunogènes a été démontré chez plusieurs espèces (lapin et hamster : Moore, 1981 ; rat : Cuasnicu et al, 1984 ; Fournier-Delpech et al, 1985). Son efficacité dépend de l'immunoréactivité de l'espèce à l'égard de l'antigène administré (Cuasnicu et al, 1990).

Nos résultats chez le bélier sont cohérents avec les observations réalisées chez le rat chez lequel on constate des altérations réversibles des fonctions spermatiques consécutives à l'iso-immunisation à l'aide de l'oPES (Fournier-Delpech et al, 1985 ; Cuasnicu et al, 1990). Les béliers traités avec l'oPES ovine pure, administrée selon différents protocoles d'immunisation, ont développé en 3 à 4 sem une asthénospermie transitoire (fig $1 \mathrm{~A}, \mathrm{~B}, \mathrm{C}$ ) qui peut être rapidement réactivée en $10 \mathrm{j}$ environ (fig 1C) par une injection de rappel ou bien maintenue pendant plusieurs semaines par la seule administraton de la protéine (fig 1D). L'administration de PES dans de l'adjuvant (Freund ou Titer Max) a généré la présence prolongée d'immunoglobulines anti-PES sériques et celle plus brève d'immunoglobines anti-PES séminales. C'est à ces dernières que peut être imputée l'asthénospermie en raison du caractère concomitant de leur présence et des altérations significatives de la mobilité spermatique chez les béliers traités, les anti-PES étant au contact de la pièce intermédiaire sur laquelle se fixe la PES (Fournier-Delpech et al, 1988). Cette hypothèse trouve un fondement dans l'immobilisation rapide $(20 \mathrm{~min}$, $37^{\circ} \mathrm{C}$ ) qui résulte de l'adjonction in vitro au milieu d'incubation (PBS) des immunoglo- 
bines sériques extraites de sérum soit lapins immunisés contre l'oPES (Fournier-Delpech et al, 1987) soit béliers traités (C et $D$ du présent travail) alors que celles de lapins non immunisés ou de béliers homologues témoins sont sans effets (Fournier-Delpech et Guérin, manuscrit en préparation).

Les titres des anti-PES sériques sont relativement faibles au maximum de la réponse biologique en raison sans doute de barrières physiologiques à l'iso-immunisation (Argawal et Hoffer, 1989). En effet, dans les conditions de sensibilité les plus élevées, la dilution des sérums n'a pas dépassé le $1 / 5000$ pour qu'une réponse fut quantifiable. La présence des anti-PES a été aussi détectée dans les sécrétions génitales mâles. Elle pose problème car, bien que le système microvasculaire du testicule du rat prépubère soit perméable aux IgG (Pollanen et Setchell, 1989), chez l'adulte les barrières anatomiques s'opposent à la transsudation dans la lumière des tubes séminifères ou le canal de l'épididyme antérieur des protéines sériques de plus de 70 kD (Argawal et Hoffer, 1989). Les immunoglobulines peuvent cependant transsuder du sérum sanguin dans les fluides des vésicules séminales et/ou de la prostate ou bien être élaborées par des structures anatomiques analogues à celles qui assurent la protection immunologique des épithéliums bronchiques et digestifs. La présence dans le plasma séminal humain d'anticorps immobilisants est connue (Koyama et al, 1991). On constate aussi chez des lapins immunisés avec de l'albumine que les spermatozoïdes portent des lgG présentes dans le fluide de la queue de l'épididyme. Cette région serait donc perméable aux immunoglobulines (Weininger et al, 1982). En revanche, l'épididyme du rat a été imperméable aux immunoglobulines anti-DE préparées chez le lapin puis administrées par la jugulire (Wong et al, 1983). Le passage des immunoglobulines dans le plasma épididymaire semble donc possible au niveau de la queue de l'épididyme selon un mécanisme encore énigmatique.

On peut penser que les anticorps antiPES bloquent l'activité biologique des PES soit parce qu'ils empêchent leur liaison aux spermatozoïdes du fait du complexe antigène-anticorps qu'ils forment au préalable, soit parce qu'ils inhibent l'activité biologique de la PES après sa liaison au site spermatique récepteur. Ces anticorps ne semblent pas cytotoxiques pour l'épithélium épididymaire car nous n'avons jamais observé de débris cellulaires dans les éjaculats des béliers traités. II faut sans doute rechercher une explication de leur effet sur les spermatozoïdes au cours de la maturation épididymaire, dans l'inhibition d'une activité biologique de l'oPES par les anti-PES puisque, chez les béliers traités, la gouttelette cytoplasmique était en position proximale comme dans le rete testis, témoignant d'un défaut de maturation dans l'épididyme. Un certain nombre de protéines épididymaires analogues à la PES sont susceptibles de provoquer une immunoasthénospermie : en particulier, les antigènes 2D6 (Jones et al, 1985) et 26 kD 4E9 (Moore et al, 1994) chez le rat, la protéine $23 \mathrm{kD}$ du hamster (Hoos et Olson, 1988), l'antigène ESA152 du bélier (Weaver et al, 1993), la protéine 135 kD du porc (Okamura et al, 1992), l'antigène SMA4 de la souris (Vernon et al, 1987), les protéines 0.31 et 0.43 de l'homme (Tézon et al, 1985 ; Sanjuro et al, 1990).

Les adjuvants de Freund ou de Hunter ont permis d'induire des réponses biologiques qui diffèrent dans le temps selon le type d'adjuvant et sa voie d'administration : l'adjuvant de Freund administré par voie intrapéritonéale provoque une réponse biologique qui semble plus rapide que l'administration intradermique dont les effets sont plus durables. Après induction de la réponse immunologique, la PES est immunogène per se au moins un certain temps (fig 1). Une conclusion analogue est rapportée chez le taureau (Finnerty et al, 1994). Les adju- 
vants seuls provoquent un effet aléatoire mis en évidence par la diminution du pourcentage de spermatozoïdes fléchants selon un processus particulier distinct de celui des anti-PES car significativement différent et n'étant pas associé à la génération d'immunoglobines anti-PES (fig 1, A, B, C) ; cet effet même s'il peut avoir une origine testiculaire (Pelletier et al, 1981) n'a pas eu de répercussion sur la spermatogenèse puisque la concentration spermatique des éjaculats a été la même chez tous les béliers.

Les résultats de fertilité sont statistiquement les mêmes après restauration d'un nombre suffisant de spermatozoïdes mobiles chez les animaux immunisés que chez les témoins. L'immunisation à l'aide de la PES ne provoque pas d'altération tératogène de la spermatogenèse ; son effet apparaît strictement post-testiculaire, probablement localisé au niveau épididymaire ou post-épididymaire.

\section{CONCLUSION}

L'iso-immunisation de béliers contre une protéine épididymaire qui se lie aux spermatozoïdes, la PES ovine, induit une importante asthénospermie transitoire. II importe de vérifier l'innocuité des anti-PES sur la descendance des béliers qui ont recouvré une mobilité spermatique suffisante pour permettre la fécondation.

Les auteurs remercient les Drs M Courot, MT de Reviers et M Bosc (PRMD, INRA, Tours-Nouzilly) et le Pr L Lewin (Clinical Biochemistry, Faculty of Medecine, Tel-Aviv) pour leurs conseils au cours de la réalisation de ce travail.

\section{RÉFÉRENCES}

Amann RP (1987) Functions of the epididymis in bulls and rams. $J$ Reprod Fert Suppl 34, 115-131
Argawal A, Hoffer A (1989) Uitrastructural studies on the development of the blood-epididymis barrier in immature rats. $J$ Androl 10, 6, 425-431

Brooks DE (1988) Androgen regulated epididymal secretory proteins associated with post-testicular development. Ann NY Acad Sci 513, 4, 179-194

Cameo MS, Dawidowski A, Gonzalez Echeverria G, Blaquier JA (1990) Changes in sperm specific antigens during epididymal maturation. In : Gamete Interaction: Prospects for Immunocontraception 1 (NJ Alexander, D Griffin, JM Spieler, G Waites, eds), Wiley Liss, Inc, 129-141

Carles C, Fournier-Delpech S, Ribadeau-Dumas B (1992) Purification of the ram epididymal androgen dependent protein «Préalbumine épididymaire spécifique (O-PES)". Evidence for strong homologies with blood albumins. Reprod Nutr Develop 32, 277 284

Cooper TG (1986) The Epididymis, Sperm maturation and Fertilisation (TG Cooper, ed), Springer Verlag, Berlin

Cuasnicu PS, Gonzalez Echeverria MF, Piazza AD, Cameo MS, Blaquier JA (1984) Antibodies against epididymal glycoproteins block fertilizing ability in the rat. J Reprod Fertil 72, 467-471

Cuasnicu PS, Conesa D, Rochwerger L (1990) Potential contraceptive use of an epididymal protein that participate in fertilization. In : Gamete Interaction: Prospects for Immunocontraception (NJ Alexander, D Griffin, JM Spieler, G Waites, eds), Wiey Liss Inc, 143-153

Dacheux JL, Dacheux F, Paquignon M (1991) Changes in sperm surface membrane and the protein content of fluid during epididymal transit in the boar. Reprod Dom Anim Suppl 1, 29-35

Finnerty M, Enright WJ, Morrison CA, Roche JF (1994) Immunization of bull calves with a $\mathrm{GnRH}$ analoguehuman serum albumin conjugate: effect of conjugate dose, type of adjuvant and booster interval on immune, endocrine, testicular and growth response. $J$ Reprod Fert 101, 333-343

Fournier-Delpech S, Courot M (1981) Glycoproteins of ram sperm plasma membrane. Relationship of protein having affinity for Con A to epididymal maturation. Biochem Biophys Res Commun 96, 756-761

Fournier-Delpech S, Courot M (1987) Sperm zona pellucida binding ability. In : Oxford Reviews of Reproductive Biology, vol 9 (J Clarke, ed), 294-321

Fournier-Delpech S, Colas G, Courot M, Ortavant R, Brice $G$ (1979) Epididymal sperm maturation in the ram: motility, fertilizing ability and embryonic development after intrauterine insemination in the ewe. Ann Biol Anim Biochem Biophys 19, 957-605

Fournier-Delpech S, Courot M, Dubois MP (1985) Decreased fertility and motility of spermatozoa from rats isoimmunized with a prealbumin epididymal specific protein. Int J Androl 6, 246-250 
Fournier-Delpech S, Holland MK, Skudlarek MD, Rankin TL, Orgebin-Crist MC, Courot M (1987) A ram epididymal secretory protein shares common antigenic determinants with rat epididymal proteins and human plasma proteins. Reprod Nutr Dev 28, 12831299

Fournier-Delpech S; Pisselet C, Garnier DH, Dubois MP, Courot M (1988) Localisation sur la tête des spermatozoïdes de bélier des sites d'affinité pour la préalbumine épididymaire androgène dépendante 64 kD. CR Acad Sci Paris Série 3293, 589-594

Fournier-Delpech S, Carles C, Ribadeau-Dumas B, Combarnous $Y$, Courot M (1990) Purification of the ram androgen dependent protein «Préalbumine epididymaire spécifique (O-PES). In : 4th Symposium on protein purification technologies" GRB, ClermontFerrand, 131-136

Garrett SH, Garrett JE, Douglas J (1991) In situ histochemical analysis of region specific gene expression in adult rat epididymis. Mol Reprod Dev 30, 1-17

Goldberg E (1990) Lactatedehydrogenase $C_{4}$ as an immunocontraceptive model. In : Gamete Interaction: Prospects for Immunocontraception, Wiley-Liss, Inc, 63-73

Hammerstedt RH, Parks JE (1987) Changes in sperm surface associated with epididymal transit. $J$ Reprod Fertil Suppl 34, 133-149

Hoos PC, Oison GE (1988) Characterization of a $23 \mathrm{kD}$ sperm-binding polypeptide of the hamster epididymis. Biol Reprod 39, 131-149

Jones R (1989) Membrane remodeling during sperm maturation in the epididymis. In : Oxford Reviews of Reproductive Biology, vol 11, 285-337

Jones R, Colin R, Brown K, Gloss VG, Gaunt SJ (1985) Development of a maturation antigen on the plasma membrane of rat spermatozoa in the epididymis and its fate during fertilization. Exp Cell Res 156, 31-44

Koyama K, Kameda K, Nakamura H, Sigheta M (1991) Recognition of carbohydrate antigen epitopes by sperm-immobilizing antibodies in sera of infertile women. Fertil Ster 56, 954-959

Moore HDM (1981) Glycoprotein secretion of rabbit and hamster epididymis. Localization on epididymal spermatozoa and the effect of specific antbodies on fertilization in vivo. J Exp Fertil 2, 77-85

Moore A, Ensrud KM, White TW, Frethem CD, Hamilton DW (1994) Rat epididymis-specific sperm maturation antigens. 1 . Evidence that the $26 \mathrm{kD} 4 \mathrm{E} 90$ antigen found on rat caudal epididymal sperm tail is derived from a protein secreted by the epididymis. Mol Reprod Dev 37, 181-194

Okamura N, Dacheux F, Venien A, Onoe, Huet JC, Dacheux JL (1992) Localization of a maturationdependent epididymal sperm surface antigen reco- gnized by a monoclonal antibody raised against a 135 kD protein in porcine epididymal fluid. Biol Reprod 47, 1040-1052

Orgebin-Crist MC, Danzo BJ, Davies J (1975) Endocrine control of development and maintenance of sperm fertilizing ability in the epididymis. In : Handbook of Physiology Sec 7, Endocrinology 5 (DW Hamilton, RO Greep, eds), American Physiol Soc, Bethesda, 319-336

Pelletier RM, Nemirovsky MZ, Calvert R, Hugon JS (1981) Effects of immunization with Freund complete adjuvant and isologous spermatozoa on the epithelium and blood-tesis barrier in guinea pig. Anat Rec 1099, 197-211

Pollanen PP, Setchell BP (1989) Microvascular permeability to $\mathrm{lgG}$ in the rat testis at puberty. Int $J$ Androl 12, 206-218

Primakoff P, Myles DG (1990) Progress toward a birth control vaccine that blocks sperm function. In : Gamete Interactions: Prospects for Immuno Contraception, Wiley Liss Inc, 89-102

Rochwerger L, Cuasnicu PS (1992) Redistribution of a rat sperm epididymal glycoprotein after in vivo capacitation. Mol Reprod Dev 31, 34-41

Sanjuro Cl, Dawidsky AR, Cameo MS, Echeverria GK, Blaquier JA (1990) Participation of human epididymal sperm epididymal sperm coating antigens in fertilization. J Androl 11, 2, 476-483

Tezon JG, Vazquez MH, Lucrecia P, De Larminat MA, Blaquier JA (1985) Identification of androgen-induced proteins in human epididymis. Biol Reprod 32, 584590

Vernon RB, Muller CH, Eddy EM (1987) Further characterization of a secreted epididymal glycoprotein in mice that binds to sperm tails. $J$ Androl $8,123-128$

Vreeburg JTM, Holland MK, Orgebin-Crist MC (1992) Binding of epididymal proteins to rat spermatozoa in vivo. Biol Reprod 47, 588-597

Weaver FE, Dino JE, Germain BJ, Wolf de, Fairbanks G (1993) Biochemical characterization of the maturation-dependent ram sperm surface antigen ESA 152. Mol Reprod Dev 35, 293-301

Weininger RB, Fisher S, Rifkin J, Bedford JM (1982) Experimental studies on the passage of specific lgG into the lumen of the rabbit epididymis. $J$ Reprod Fert 66, 251-258

Wong PYD, Tsang AYF, Lau HK (1983) Restricted entry of an anti-rat epididymal protein Igg ineo the rat epididymis. Int $J$ Androl 6, 275-282

Yanagimachi R, Okada A, Tung KSK (1981) Sperm autoantigens and fertilization effects of anti-guinea pig sperm auto-antibodies on sperm ovum interactions. Biol Reprod 24, 512-518 